\title{
Pre-print journal club review of BioRxiv article: Uncovering the hidden antibiotic potential of Cannabis
}

\author{
Craig McCormick ${ }^{1}$ \\ ${ }^{1}$ Dalhousie University
}

May 5, 2020

We, the students of MICI5029/5049, a Graduate Level Molecular Pathogenesis Journal Club at Dalhousie University in Halifax, NS, Canada, hereby submit a review of the following BioRxiv preprint:

Maya A. Farha, Omar M. El-Halfawy; Robert T. Gale; Craig R. MacNair; Lindsey A. Carfrae; Xiong Zhang, Nicholas G. Jentsch; Jakob Magolan; Eric D. Brown. Uncovering the hidden antibiotic potential of Cannabis. BioRxiv 833392; doi:http://dx.doi.org/10.1101/833392

We will adhere to the Universal Principled (UP) Review guidelines proposed in:

Universal Principled Review: A Community-Driven Method to Improve Peer Review.Krummel M, Blish C, Kuhns M, Cadwell K, Oberst A, Goldrath A, Ansel KM, Chi H, O'Connell R, Wherry EJ, Pepper M; Future Immunology Consortium. Cell . 2019 Dec 12;179(7):1441-1445. doi: 10.1016/j.cell .2019.11.029.

SUMMARY : In this study, Farha, et al . investigate candidate antibiotics from Cannabis sativa . Eighteen molecules from C. sativa were screened for antibiotic activity against methicillin-resistant Staphylococcus aureus (MRSA). Of these, seven compounds were identified as potential antibiotics (min. inhibitory concentration $[\mathrm{MIC}]<2 \mu \mathrm{g} / \mathrm{mL}$ ). The cannabinoid cannabigerol $(\mathrm{CBG})$ displayed the highest efficacy against biofilms, free-living MRSA, and MRSA persister cells. The authors used a variety of approaches to investigate $\mathrm{CBG}$ mechanism of action, including attempts to isolate drug-resistant bacteria via spontaneous resistance, serial passaging, knockdown libraries and transposon mutant libraries. They were unable to identify a single CBG-resistant bacterium. However, they determined that CBG affects the membrane stability of Gram-positive bacteria. Finally, they found that CBG alone had no effect on Gram-negative bacteria, but displayed antibiotic activity when combined with polymyxin B. Overall, this paper shows that there are multiple metabolites produced by Cannabis sativa that have antibacterial properties and that CBG can kill bacterial cells and disrupt bacterial biofilms by interfering with stability of the inner membrane.

\section{OVERALL ASSESSMENT:}

STRENGTHS: The authors showed that several metabolites from C. sativa had MIC values that warranted further investigation as candidate antibiotics. They not only assessed killing of free-living MRSA, but they extended the study to examine biofilm disruption and killing of persister cells, increasing the clinical relevance of the study. Furthermore, the authors investigated how readily MRSA can acquire resistance to CBG, one of the seven metabolites they identified in their screen. They could not identify a single resistant mutant through a variety of different screening approaches, indicating that resistance could be difficult to develop. Finally, the authors determined that CBG works by disrupting the cell membrane of Gram-positive bacteria. They found that CBG alone could not kill Gram-negative bacteria but disrupting the outer membrane with polymyxin B allowed CBG to work on the inner membrane and restore its antibacterial effects. Overall, the study has clear potential for impact and provides a foundation for future studies.

WEAKNESSES: While the experimental design was generally good, we identified some weaknesses in data 
presentation. The methods section was lacking in detail required for others to reproduce experiments. There were inconsistencies in figure labels and legends that must be addressed. While the authors showed that CBG is an effective antibiotic, it was not clear whether this molecule has any toxicity in animals or humans that would limit its utility. This could be addressed by discussing previous CBG studies that established these parameters, or through in vivo experiments. Finally, the in vivo aspect of this study was underdeveloped; we believe that this study could have benefited from evaluating the effectiveness of polymyxin B and CBG in a skin infection model, as polymyxin B is known to be toxic if ingested (there was some debate in the group as to whether this skin model was beyond the scope of the current manuscript). Without more in vivodata or information about $\mathrm{CBG}$ toxicity it remains difficult to properly evaluate the manuscript.

\section{DETAILED U.P. REVIEW ASSESSMENT: OBJECTIVE CRITERIA (QUALITY)}

Quality: Experiments (1-3 scale) SCORE $=2$ (hard to determine based on incomplete Materials \& Methods section)

Figure by figure, do experiments, as performed, have the proper controls?

Figure 2: We found that Figure 2B was missing a control $0 \mu \mathrm{g} / \mathrm{mL}$ dose. Figure $2 \mathrm{C}$ had a spike in biofilm formation at $2 \mu \mathrm{g} / \mathrm{mL}$ and it is unclear whether this is a true biological event or technical issue. It is unclear whether the " $\mathrm{n}$ " in this experiment represents individual biological replicates or one experiment with eight technical replicates. The authors should clarify this point. Figure 2D: While it was nice to see a negative control in this experiment it was a positive control; NH125 is commercially available has been previously proven to kill MRSA persister cells (https://www.ncbi.nlm.nih.gov/pmc/articles/PMC4976882/). Figure 3: It was not clear how cell growth rates were normalized in the Tn mutant library screen. In general, further methodological details on these experiments is required. Figure 4: We could not read this figure due to poor resolution although we were curious as to why there was a differing number of mice in the vancomycin control and vehicle control groups. Figure 5: Labelling on Figure 5D did not explain why there were three different heat maps for each group of bacteria tested. Further details on this figure are required to aid reader comprehension.

Are specific analyses performed using methods that are consistent with answering the specific question?

While difficult to assess this due to insufficient information in the Materials \& Methods, we believe that the analyses performed were consistent with other studies in the field.

Is there the appropriate technical expertise in the collection and analysis of data presented?

We identified multiple deficiencies in data presentation in this paper:

- Axis labelling between Figure 2B and 2C were inconsistent.

- Figure 3 lacked details in general and would benefit from added detail in the figure legend.

- We were unable to read Figure 4 (low resolution).

- Labelling in Figure 5 did not describe what the three different heatmaps represented.

- Figure 1 could be improved by presenting the MIC curves for each compound alongside its structure.

Do analyses use the best-possible (most unambiguous) available methods quantified via appropriate statistical comparisons?

We did not identify any deficiencies in statistical analyses, but the authors should explain why a different number of mice were used in Figure 4.

Are controls or experimental foundations consistent with established findings in the field? A review that raises concerns regarding inconsistency with widely reproduced observations should list at least two examples in the literature of such results. Addressing this question may occasionally require a supplemental figure that, for example, re-graphs multi-axis data from the primary 
figure using established axes or gating strategies to demonstrate how results in this paper line up with established understandings. It should not be necessary to defend exactly why these may be different from established truths, although doing so may increase the impact of the study and discussion of discrepancies is an important aspect of scholarship.

Experiments and controls are consistent with other studies in the field that show that Cannabis sativa has antimicrobial properties.

Quality: Completeness (1-3 scale) SCORE $=1.5$

Does the collection of experiments and associated analysis of data support the proposed title- and abstract-level conclusions? Typically, the major (title- or abstract-level) conclusions are expected to be supported by at least two experimental systems.

The experiments do not adequately support the conclusions stated in the abstract. The title is quite ambiguous.

Are there experiments or analyses that have not been performed but if "true" would disprove the conclusion (sometimes considered a fatal flaw in the study)? In some cases, a reviewer may propose an alternative conclusion and abstract that is clearly defensible with the experiments as presented, and one solution to "completeness" here should always be to temper an abstract or remove a conclusion and to discuss this alternative in the discussion section.

- While there are no experiments that come to mind that would disprove the conclusions of this paper, we believe that a skin infection model experiment would add significant value as polymyxin B and CBG could be used as a topical agent against both Gram-positive and Gram-negative bacterial infections.

- We noticed that in supplemental Figure S4C a number of genes involved in vitamin biosynthesis and metabolic processes were also enriched. We would like to see this added to the discussion as it could suggest an alternative mode of action. Despite this, we remain convinced that at least one of CBG's modes of action was disruption of cytoplasmic membranes.

Quality: Reproducibility (1-3 scale) SCORE $=3$

Figure by figure, were experiments repeated per a standard of 3 repeats or 5 mice per cohort, etc.?

As mentioned above, it is not clear whether the sample size (n) for experiments in Figure 2 represent a single experiment with multiple replicates or multiple different experiments.

Is there sufficient raw data presented to assess rigor of the analysis?

Yes, we believe enough raw data is presented.

Are methods for experimentation and analysis adequately outlined to permit reproducibility?

- The methods section is lacking in detail required to reproduce the results. A few specific examples are pointed out below but in general almost all experiments require further clarification re: the methods.

- Experimental methods for the MIC 90 experiment were not included in the methods at all. This should be addressed.

- The analysis of the resistant mutant data was lacking in detail. More detailed information would aid understanding of how the authors concluded that there was not a single mutant that was sensitized to sublethal concentrations of CBG.

- Additional information is required to understand how the authors obtained persister cells.

Quality: Scholarship (1-4 scale but generally not the basis for acceptance or rejection) SCORE $=2$

Has the author cited and discussed the merits of the relevant data that would argue against their conclusion?

- For the most part, the authors cite other work appropriately. The novelty of the first screen for antibiotic potential is unclear. It would be nice to clarify this issue of novelty in the 
text, as we found a previous study that identified antibiotic potential of whole plant extracts (https://doi.org/10.1016/j.joim.2018.07.005).

- We think that the paper would benefit from further discussion on the how these metabolites may protect the Cannabis sativa plant. Elaboration on whether bacterial infection is common in this plant and what bacteria are known to infect this plant may help give us an idea of the role of CBG in its natural context.

Has the author cited and/or discussed the important works that are consistent with their conclusion and that a reader should be especially familiar when considering the work?

Yes, although their discussion on CBG as a natural defense against plant pathogens could be broadened.

Specific (helpful) comments on grammar, diction, paper structure, or data presentation (e.g., change a graph style or color scheme) go in this section, but scores in this area should not to be significant bases for decisions.

- As mentioned previously, multiple low-resolution figures need to be recreated as they are currently difficult to interpret.

- The prose could be improved.

- We found a few typos that should be addressed including:

- ".." in line 219

- And "fig.2a in line 146 instead of "fig. 2a"

\section{MORE SUBJECTIVE CRITERIA (IMPACT)}

Impact: Novelty/Fundamental and Broad Interest (1-4 scale) SCORE $=2$

A score here should be accompanied by a statement delineating the most interesting and/or important conceptual finding(s), as they stand right now with the current scope of the paper. A "1" would be expected to be understood for the importance by a layperson but would also be of top interest (have lasting impact) on the field.

- The authors show that multiple C. sativa metabolites have promising antibiotic potential. Furthermore, they show that CBG specific is able to destroy biofilms and kill persister cells by impacting the inner membrane stability of bacteria in an unknown mechanism. Interestingly, they were unable to find a single knockout or knockdown that all bacteria to become resistant to CBG effects. This suggests that there may be a high genetic barrier to the emergence of drug resistance. Finally, they were able to show that permeabilizing/disturbing the outer membrane of Gram-negative bacteria rendered these organisms sensitive to the effect of CBG. This is important because we desperately need new antibiotics to target Gram-negative bacterial pathogens.

- Due to the high likelihood of a large public interest in this work we would like to stress the importance of a candid discussion of the limitations of the study in the Discussion.

How big of an advance would you consider the findings to be if fully supported but not extended? It would be appropriate to cite literature to provide context for evaluating the advance. However, great care must be taken to avoid exaggerating what is known comparing these findings to the current dogma (see Box 2). Citations (figure by figure) are essential here.

Yes, there was general agreement that this paper does provide a significant advancement in this area of research without the requirement of further experiments.

Impact: Extensibility (1-4 or N/A scale) SCORE $=3$

Has an initial result (e.g., of a paradigm in a cell line) been extended to be shown (or implicated) to be important in a bigger scheme (e.g., in animals or in a human cohort)? This criterion is only valuable as a scoring parameter if it is present, indicated by the N/A option if it simply doesn't apply. The extent to which this is necessary for a result to be considered of value is 
important. It should be explicitly discussed by a reviewer why it would be required. What work (scope and expected time) and/or discussion would improve this score, and what would this improvement add to the conclusions of the study? Care should be taken to avoid casually suggesting experiments of great cost (e.g., "repeat a mouse-based experiment in humans") and difficulty that merely confirm but do not extend (see Bad Behaviors, Box 2).

- The authors demonstrate that their compounds of interest could have clinical applications through the mouse model experiment. However, the toxicity of the compounds from both acute and chronic use was not addressed. This is a major deficiency in the study because many compounds can kill bacterial cells but are not considered useful antibiotics due to their toxic effect on the host. To address this issue, we suggest that the authors increase the duration of their animal experiments. However, we understand that this may not be possible, in which case we would like to see a discussion of the current literature regarding whether the dosage of CBG in other drugs is well-tolerated and to what extent it has been used in humans.

- We believe that the implications from this study could be extended by using a skin infection model in combination with polymyxin B. This would indicate whether CBG may be a good candidate for use as a topical antibiotic for both Gram positive and Gram-negative bacteria.

- We believe that the extensibility of this work could have been increased dramatically within vivo experiments that show CBG's ability to work in multiple different infection models. Furthermore, they could have benefited greatly from an in vivo model showing the synergy between polymyxin B and CBG. 